\title{
Revisão Narrativa sobre Choque na Sala de Emergência
}

\section{A Narrative Review on Shock in the Emergency Room}

Jorge DANTAS $₫ 1$, Rita VAZ², Rui MORAIS ${ }^{3}$, Irene VERDASCA ${ }^{4}$

Acta Med Port 2021 Jun;34(6):451-459 - https://doi.org/10.20344/amp.11704

RESUMO

O doente em choque representa um desafio clínico comum na sala de emergência, e representa uma potencial situação de risco de vida imediato que requer a intervenção de diferentes especialidades médicas na sua estabilização. Neste trabalho realiza-se uma revisão não sistemática de recomendações internacionais e de literatura adicional sobre este tema, sob a perspectiva da abordagem ao doente na sala de emergência e das particularidades deste contexto. Esta revisão é estruturada segundo uma proposta de protocolo de actuação baseada no diagnóstico precoce do quadro de choque, na identificação do tipo de choque e da etiologia mais provável bem como na instituição de terapêutica de suporte. É destacada a importância da estabilização inicial do doente ser orientada pela identificação dos mecanismos fisiológicos em falência, e identificadas estratégias que, fazendo pouco sentido noutros contextos, podem ter interesse na sala de emergência - dado serem práticas e de rápida implementação - como é o caso da administração de fármacos vasoactivos por via periférica ou em bólus.

Palavras-chave: Choque/diagnóstico; Choque/etiologia; Choque/tratamento; Serviço de Urgência Hospitalar

\section{ABSTRACT}

The patient in shock represents a common clinical challenge in the emergency room, and potentially represents an immediate lifethreatening situation that requires the intervention of different medical specialties in order for hemodynamic stabilization to be achieved This paper reports a non-systematic review of the initial approach to the patient in shock, highlighting the adaptation of the instituted measures to the particularities of the emergency room environment. This review is structured according to a proposed protocol of action based on the early diagnosis of shock, the identification of the type of shock and the most likely cause, and the institution of supportive therapy. The initial stabilization of the patient should be guided by the identification of failing physiological mechanisms, having in mind that strategies with little meaning in other contexts may be of interest in the emergency room - as they are practical and can be rapidly implemented - as is the case with the administration of vasoactive drugs in bolus or by peripheral access.

Keywords: Emergency Service, Hospital; Shock/diagnosis; Shock/etiology; Shock/therapy

\section{INTRODUÇÃO}

A Medicina de Emergência, no que se refere à patologia médica, requer a aplicação de conhecimentos comuns à Medicina Intensiva e à Medicina Interna num contexto muito próprio onde por vezes as estratégias habituais nestes ambientes não são aplicáveis directamente: Problemas como a qualidade da informação disponível (frequentemente escassa ou contraditória), ou a necessidade de instituir medidas de suporte de forma imediata (condicionadas pelo factor tempo), podem atrasar a prestação de cuidados e comprometer o prognóstico do doente.

Este aspecto é também verdadeiro para a patologia cirúrgica / traumática quando abordado em contexto da sala de emergência, já que quer a necessidade de uma abordagem multidisciplinar, quer as limitações impostas por este ambiente, são semelhantes.

Sendo uma entidade clínica relativamente comum no serviço de urgência, o choque apresenta elevada mortalidade. $^{1}$ São exemplos a sépsis, em que a mortalidade do doente em choque é de $40 \%-50 \%$ por oposição a $25 \%$ - $30 \%$ no doente sem choque ${ }^{2-4}$; ou o enfarte agudo do miocárdio em que 5\% - 15\% dos doentes evoluem para choque cardiogénico com uma mortalidade de $40 \%-50 \% .^{5}$ Está adicionalmente bem definida a importância para o prognóstico de uma identificação e atuação precoces neste contexto. ${ }^{1-5}$

O presente trabalho tem como objectivo rever de modo não sistemático a abordagem inicial ao doente adulto em choque realizada na sala de emergência.

\section{MATERIAL E MÉTODOS}

Foi feita uma análise das recomendações internacionais das sociedades europeias de Cuidados Intensivos, de Cardiologia, e do European Resuscitation Council. Foi seleccionada ainda bibliografia adicional, com relevância para a abordagem ao doente em choque em contexto da sala de emergência - recorrendo a pesquisa na PubMed pelas seguintes palavras-chave: 'shock', 'distributive', 'septic', 'cardiogenic', 'hypovolemic', 'hemorrhagic', 'obstructive', 'fluids', 'vasopressor', 'noradrenaline', 'norepinephrine', 'push-dose', 'bolus-dose', 'heart failure', 'myocardial infarction', 'peripheral intravenous', 'sympathomimetics', 'emergency department', 'emergency room', e 'physiology'. Foi também

1. Unidade de Cuidados Intensivos Polivalente. Hospital Curry Cabral. Centro Hospitalar de Lisboa Central. Lisboa. Portugal.

2. Zoorgsaam. Hulst. Países Baixos.

3. Unidade de Cuidados Intensivos Polivalente. Hospital São Francisco Xavier. Centro Hospitalar de Lisboa Ocidental. Lisboa. Portugal.

4. Serviço de Medicina Interna. Hospital São Francisco Xavier. Centro Hospitalar de Lisboa Ocidental. Lisboa. Portugal.

$\triangle$ Autor correspondente: Jorge Dantas. jorgeoliveiradantas@gmail.com

Recebido: 17 de dezembro de 2018 - Aceite: 21 de outubro de 2019 - First published: 16 de abril de 2021 - Online issue published: 01 de junho de 2021 Copyright (C) Ordem dos Médicos 2021 
revisto o protocolo de transfusão maciça da Direcção Geral de Saúde da República Portuguesa, dada a sua relevância para a abordagem inicial do doente em choque hemorrágico.

A opinião dos autores sobre os temas abordados encontra-se expressa na discussão do artigo.

A estrutura do presente trabalho encontra-se desenhada de acordo com o braço esquerdo da proposta de protocolo de actuação que se apresenta na Fig. 1.

Assumindo que todos os doentes com instabilidade clínica aparente, e que não se encontram em paragem cardiorrespiratória, devem ser abordados segundo a metodologia airway, breathing, circulation, disability, exposure (AB$\mathrm{CDE}){ }^{6,20}$ a presente revisão foca-se na abordagem de $C$ (circulation) no contexto da sala de emergência, e parte daí para o diagnóstico de choque, para a identificação do tipo de choque e para a identificação da etiologia mais provável em cada situação clínica. É apresentada posteriormente a revisão da terapêutica inicial de suporte.

Não é revista neste artigo a terapêutica dirigida em es- pecífico a cada etiologia.

A Fig. 2 sublinha os elementos clínicos que devem alertar precocemente para um possível diagnóstico de choque, complementando a informação apresentada na Fig. 1.

No mesmo sentido, a Fig. 3 assinala três factores que devem ser avaliados - débito cardíaco, estado volémico e tónus vascular - uma vez que, em conjunto com os restantes dados clínicos recolhidos, facilitam a distinção entre os vários tipos de choque e podem orientar a marcha diagnóstica para etiologias em específico.

Concomitantemente, já que de acordo com a metodologia $A B C D E$ estes passos devem ser realizados praticamente em simultâneo, ${ }^{6,20}$ são também destacados os grupos principais da terapêutica inicial de suporte.

\section{Definição de choque}

O consenso de 2014 da Sociedade Europeia de Cuidados Intensivos define o choque como uma falência circulatória aguda, generalizada e potencialmente fatal, associada a utilização inadequada de oxigénio a nível celular. ${ }^{1}$

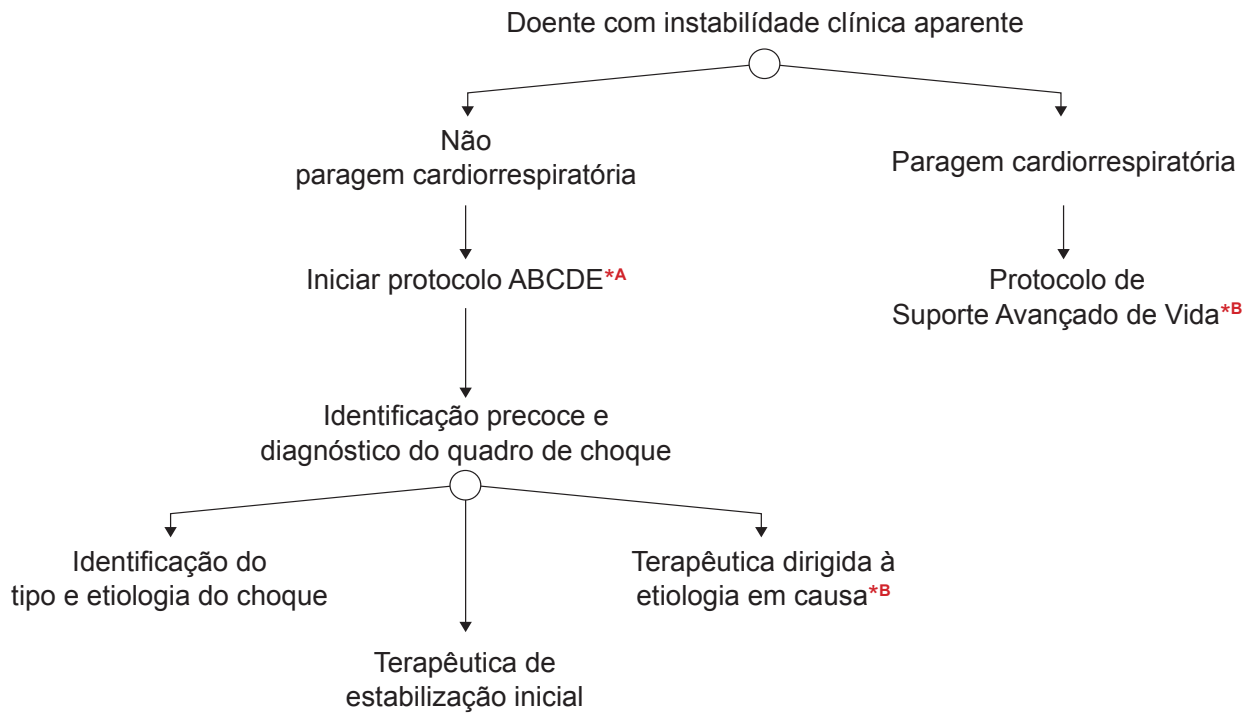

Figura 1 - Proposta de protocolo de actuação

*A: É focado neste artigo fundamentalmente a abordagem de $C$ (circulation) no que se refere à metodologia $A B C D E$, não sendo revista esta metodologia no seu todo. ${ }^{*}$ B: Temas não revistos neste artigo.

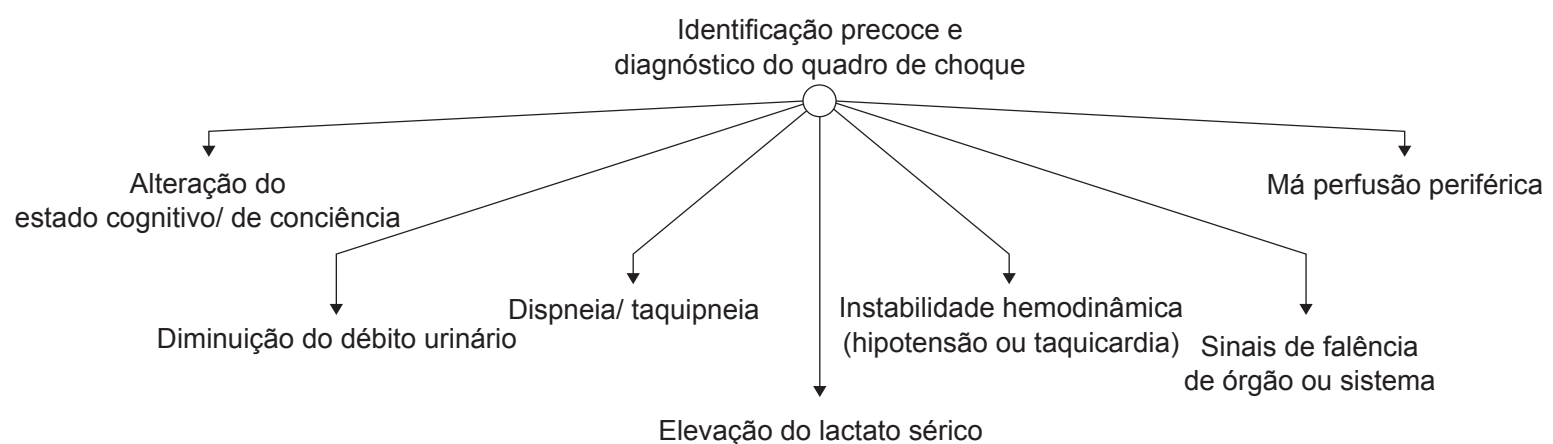

Figura 2 - Elementos clínicos que devem levantar a suspeita de um quadro de choque 


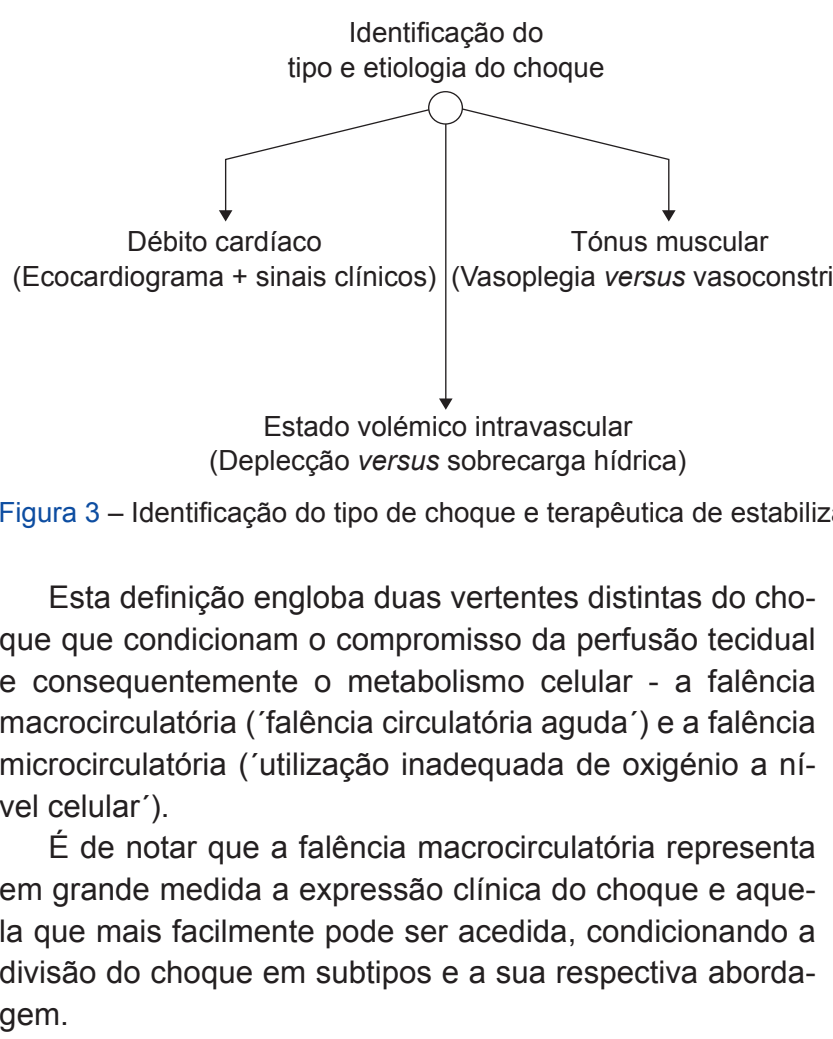

\section{Diagnóstico de choque}

É de considerar o diagnóstico de choque quando um doente apresenta sinais de compromisso macrovascular ou microvascular com consequente desajuste da entrega celular de $\mathrm{O}_{2}{ }^{1}$

São sinais e sintomas deste compromisso: alteração do estado cognitivo/de consciência, dispneia/taquipneia, instabilidade hemodinâmica (hipotensão ou taquicardia), má perfusão periférica, diminuição do débito urinário e elevação do lactato sérico, bem como sinais de falência de outros órgãos ou sistemas.

Sendo composto por uma síndrome muito inespecífica, mas que apresenta elevada mortalidade e morbilidade, é necessário um alto índice de suspeição de forma a que este diagnóstico e a etiologia na sua origem sejam activamente pesquisados. ${ }^{1-4}$

\section{Reconhecimento precoce do quadro de choque}

A abordagem inicial a um doente que aparente instabilidade clínica e não se encontre em paragem cardiorrespiratória deve seguir a abordagem $A B C D E .{ }^{1,6}$ Estando amplamente estabelecido que o reconhecimento precoce do doente em choque contribui para outcomes mais favoráveis, ${ }^{1-5}$ estes doentes devem ser avaliados segundo esta metodologia e segundo a sua filosofia de 'reconhecer e tratar'.'

Podendo os doentes em choque apresentar alterações nas diversas componentes do exame $A B C D E$, os sinais e sintomas que permitirão o diagnóstico e a definição do tipo e da etiologia de choque encontrar-se-ão maioritariamente em $C,{ }^{1}$ destacando-se os que permitem inferir sobre débito cardíaco, tónus vascular periférico (vasoconstrição versus

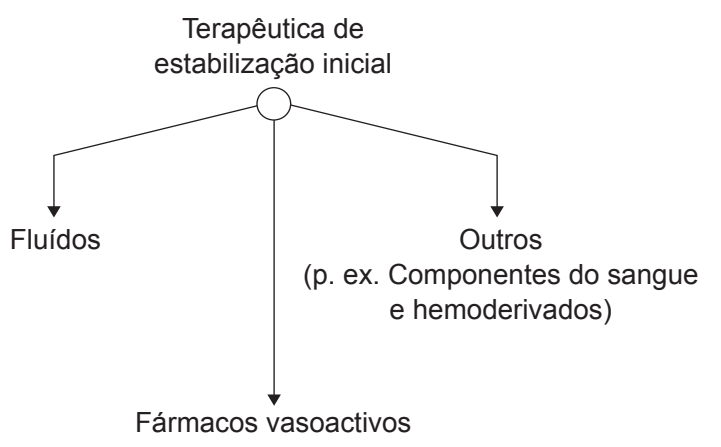

vasoplegia) e estado volémico intravascular (deplecção versus sobrecarga).

Neste sentido é importante recordar que:

- A entrega de oxigénio a nível celular $\left(\mathrm{DO}_{2}\right)$, depende directamente do débito cardíaco (DC), do valor de hemoglobina sérico $(\mathrm{Hb})$, da saturação arterial de oxigénio $\left(\mathrm{SO}_{2}\right)$, e em pequena percentagem da pressão parcial de oxigénio no plasma $\left(\mathrm{PaO}_{2}\right)$; o que se traduz pela seguinte equação: $\mathrm{DO}_{2}=\mathrm{DC} \times\left[\left(1,39 \times \mathrm{Hb} \times \mathrm{SO}_{2}\right)+\left(\mathrm{PaO}_{2} \times 0,03\right)\right]$

- O débito cardíaco é igual ao produto do volume sistólico pela frequência cardíaca;

- O volume sistólico varia directamente com a pré-carga e com o inotropismo e indirectamente com a pós-carga;

- A tensão arterial (TA) é igual ao produto do débito cardíaco pelas resistências vasculares.

Assim, caso exista um compromisso do débito cardíaco ou da entrega de $\mathrm{O}_{2}$ a nível celular, e os sistemas de compensação se mantenham patentes, é de esperar que o sistema nervoso autónomo despolete uma resposta pautada pela taquicardia, vasoconstrição periférica e taquipneia, apoiada por um conjunto de mediadores neuro-hormonais.

É também importante recordar que, no contexto de disfunção microcirculatória, a entrega de oxigénio $\left(\mathrm{O}_{2}\right)$ aos tecidos se encontra comprometida adicionalmente por incapacidade de extração a nível celular. Tal facto assume principal relevância no choque séptico, por resposta do organismo à inflamação. ${ }^{2-4}$

\section{Tipos de choque}

A integração dos diferentes tipos de choque com as etiologias mais comuns, com a respectiva apresentação clínica, e com a terapêutica inicial de suporte a considerar, encontra-se descrita sumariamente na Tabela 1, de acordo com o que é descrito no corpo do artigo.

O mecanismo fisiopatológico na origem da falência macrocirculatória é habitualmente utilizado para definir os diversos tipos de choque, uma vez que é esta 'falência circulatória aguda' que mais contribui para a apresentação clínica do doente em choque':

Choque hipovolémico: Tem origem numa diminuição do retorno venoso, por perda externa ou interna de fluido, sendo exemplos a desidratação extrema e a hemorragia major. $^{1}$ 


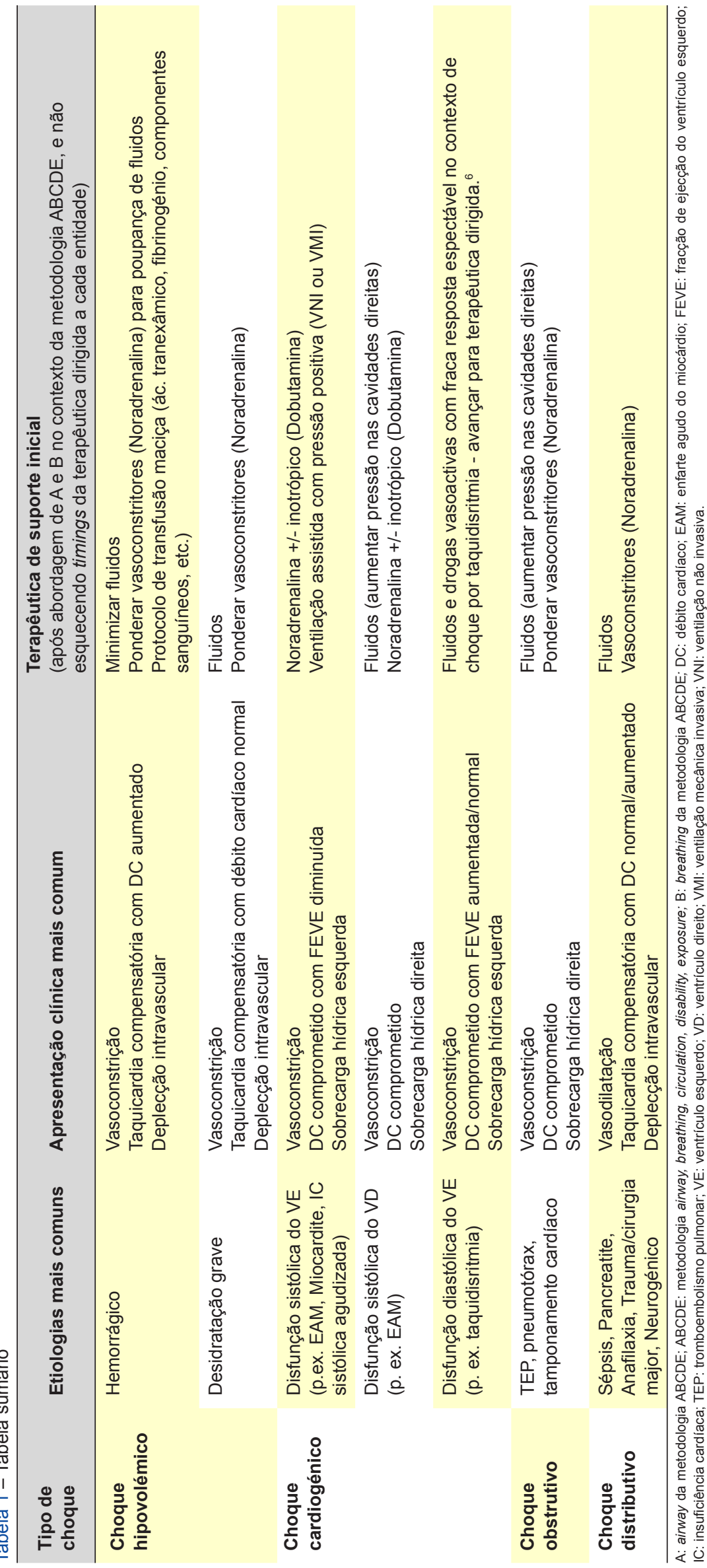

Choque cardiogénico: Depende de uma falência da bomba cardíaca, quer por compromisso da sua função sistólica em contexto de perda de contractilidade do miocárdio, quer por disfunção diastólica com compromisso do enchimento ventricular durante a diástole. Pode ainda ter origem na quebra do volume sistólico, e consequentemente do débito cardíaco por mecanismos mecânicos (ex. rutura do miocárdio ou falência valvular) ou por bradidisritmia. ${ }^{1}$

Choque obstrutivo: É provocado por uma obstrução súbita do fluxo sanguíneo, como são exemplos o tromboembolismo pulmonar, o pneumotórax hipertensivo ou o tamponamento cardíaco. ${ }^{1}$

Choque distributivo: Tem origem na resposta do organismo à inflamação ou a lesão directa do sistema nervoso central, sendo pautado por perda do tónus vascular (vasoplegia). Pode surgir no contexto de sépsis, inflamação sem infeção (como são exemplos o pós-operatório de cirurgia major, o trauma, a queimadura de uma percentagem importante da superfície corporal e a pancreatite aguda), anafilaxia e/ou intoxicação. ${ }^{1}$

É ainda de ressalvar que pode existir sobreposição dos diferentes tipos de choque por concorrência de diferentes etiologias. $^{1}$

\section{Etiologia do choque}

Tendo em conta a história e a apresentação clínica do doente, será possível determinar a etiologia mais provável para o quadro de choque. Neste sentido, importa recordar os seguintes factores, que podem contribuir para o diagnóstico diferencial:

Choque cardiogénico por enfarte agudo do miocárdio (EAM): dor precordial típica, factores de risco cardiovasculares, sinais de isquémia no electrocardiograma, alterações da contractilidade segmentar no ETT, elevação dos marcadores de necrose miocárdica. . $^{9,10}$

Choque cardiogénico por dissecção da aorta: dor precordial com irradiação ao dorso e extensão progressiva ao abdómen, pulsos assimétricos, identificação da dissecção em ETT ou angiografia por tomografia computorizada (angio-TC) torácica. O ECG poderá apresentar sinais de isquémia se a dissecção se estender à raiz da aorta. ${ }^{1,5}$ 
O choque cardiogénico pode ainda ter como etiologia: disritmias com compromisso do débito cardíaco (tanto bradi como taquidisritmias); complicações valvulares agudas/ agudizadas, insuficiência cardíaca previamente conhecida, ou patologia aguda com compromisso da função sistólica, como são exemplos as miocardites. ${ }^{7}$

Choque obstrutivo por tamponamento cardíaco: pulso paradoxal, disfunção diastólica/colapso das cavidades direitas no ETT. ${ }^{1}$

Choque obstrutivo por tromboembolismo pulmonar: factores de risco para embolia/trombose venosa, hipoxémia, polipneia, ausência de alterações na auscultação pulmonar, dilatação das cavidades direitas no ETT, elevação dos produtos de degradação da trombina (d-dímeros), trombo visível em angio-TC. ${ }^{11}$

Choque obstrutivo por pneumotórax hipertensivo: desvio da traqueia, movimentos respiratórios assimétricos, abolição do murmúrio vesicular unilateralmente. ${ }^{1}$

Choque hipovolémico por hemorragia maciça: colecções hemáticas na ecografia abdominal em contexto de trauma, história de hemorragia digestiva alta comprovada em endoscopia digestiva alta (o choque em quadros de hemorragia digestiva baixa não é comum). ${ }^{1}$

O choque hipovolémico pode também ocorrer em situações em que ocorra uma perda de líquido intravascular muito significativa, como são exemplos a desidratação grave por vómitos/diarreia, ou a transudação em caso de queimadura de uma percentagem significativa da superfície corporal. ${ }^{1}$

Choque distributivo: evidência de infecção no contexto de choque séptico; história de reacção alérgica prévia a choque anafilático; lesão do tecido nervoso no contexto de choque neurogénico; estado pró-inflamatório sem infecção. ${ }^{1-4}$

\section{Apresentação clínica}

Os sinais e sintomas de choque consistem na expressão clínica das falências microcirculatória e macrocirculatória, ${ }^{1-5}$ sendo que a diferenciação entre os diversos tipos de choque depende da expressão da falência macrocirculatória. ${ }^{1}$ Assim, é de considerar a avaliação do débito cardíaco, tónus vascular (vasoplegia versus vasoconstrição) e estado volémico (deplecção versus sinais de sobrecarga hídrica). ${ }^{2-5,7} \mathrm{Em}$ contexto de sala de emergência esta não é uma avaliação fácil, sendo de grande importância o apoio da ecocardiografia à cabeceira. ${ }^{1-5,7,8}$ No entanto, é de notar que habitualmente:

- Um doente com débito cardíaco mantido ou aumentado e défice de preenchimento vascular apresentará provavelmente um choque distributivo se se encontrar vasoplégico, ou um choque hipovolémico se se encontrar vasoconstrito.

- Um doente com débito cardíaco mantido, défice de preenchimento vascular e sinais de vasoplegia, pode apresentar no exame objectivo sinais compatíveis com sobrecarga hídrica esquerda, que neste contexto corresponderão provavelmente a um qua- dro de síndrome de dificuldade respiratória aguda e não a patologia cardíaca primária.

- Um doente com compromisso do débito cardíaco apresentará sempre vasoconstrição reactiva a menos que ocorra falência dos mecanismos de compensação.

- Um doente com débito cardíaco comprometido e sinais de sobrecarga hídrica direita apresentará na sua origem uma etiologia pautada pelo aumento da pressão no interior das cavidades direitas, como são exemplos o choque obstrutivo, o choque cardiogénico por disfunção sistólica do ventrículo direito ou por bradidisritmia. Adicionalmente, doentes com cor pulmonale ou sob ventilação com pressão positiva podem apresentar estes sinais.

- Um doente com compromisso do débito cardíaco e sinais de sobrecarga hídrica esquerda apresentará provavelmente um quadro de choque cardiogénico por disfunção sistólica do ventrículo esquerdo ou por taquidisritmia.

Estes mecanismos fisiopatológicos (débito cardíaco, tónus vascular e preenchimento vascular/sobrecarga hídrica) traduzir-se-ão em sinais e sintomas que podem ser reconhecidos numa primeira avaliação do doente segundo a metodologia airway, breathing, circulation, disability, exposure (ABCDE). ${ }^{1,6}$ Poderão ainda fazer-se uso dos meios complementares de diagnóstico disponíveis em contexto de urgência à cabeceira do doente [avaliação laboratorial, gasimetria arterial/venosa central, RX tórax, electrocardiograma (ECG), ecocardiograma transtorácico (ETT) e ecografia torácica/abdominal, etc.] que auxiliarão na avaliação do doente. Dos sinais mais relevantes destacam-se:

- O compromisso do débito cardíaco, que se traduz clinicamente na alteração do estado de consciência/ cognitivo, má perfusão periférica e diminuição do débito urinário; traduzindo-se por diminuição relativa da tensão arterial sistólica (TAS), pressão de pulso (TAS-TA diastólica) diminuída (<30-40 mmHg), disfunção sistólica do ventrículo esquerdo (VE) ou do ventrículo direito (VD) no ETT, saturação venosa central de $\mathrm{O}_{2}$ medida na veia cava superior $<70 \%$, gap de dióxido de carbono $\left(\mathrm{CO}_{2}\right)$ (pressão parcial de $\mathrm{CO}_{2}\left(\mathrm{pCO}_{2}\right)$ venoso central - $\mathrm{pCO} 2$ arterial) $>6$ $\mathrm{mmHg}$ e ainda diminuição acentuada do end tidal $\mathrm{CO}_{2}\left(\mathrm{EtCO}_{2}\right)$ quando comparada com o $\mathrm{pCO}_{2}{ }^{5}$

- Extremidades quentes, boa perfusão periférica, pressão de pulso aumentada (> 40 -50 mmHg), diminuição relativa da tensão arterial diastólica e indícios de depleção intravascular (relativa, por aumento do continente vascular) são sinais de vasoplegia. $^{2-4}$

- A vasoconstrição é sugerida por extremidades frias, má perfusão periférica, pressão de pulso diminuída (<30 - $40 \mathrm{mmHg}$ ) e aumento relativo da tensão arterial diastólica. ${ }^{5}$

- A sobrecarga hídrica esquerda é sugerida por alterações simétricas na auscultação pulmonar 
(fervores/crepitações, broncoespamo), hipoxémia, polipneia, alterações simétricas na radiografia do tórax (hipotransparência difusa bilateral) ou na ecografia pulmonar (padrão de linhas B bilateral). 5,7-8

- O ingurgitamento venoso jugular, o refluxo hepato-jugular, o edema periférico de declive e a pele húmida e suada, bem como uma veia cava inferior (VCI) ectasiada e sem variação com o ciclo respiratório no ETT e uma pressão venosa central $(P V C)>20$ $\mathrm{mmHg}$ são sinais de sobrecarga hídrica direita. $5,7,8$

- O défice de preenchimento vascular é sugerido por desidratação, sinais/história de hemorragia, uma PVC baixa e uma VCl com colapso durante o ciclo respiratório ou um VE hiperdinâmico com sinal de kissing walls (fracção de ejecção aumentada com colapso ventricular) no ETT. ${ }^{8}$

- Dada a possibilidade de realização de ecografia à cabeceira do doente, esta assume um papel de destaque de entre os diversos meios auxiliares de diagnóstico. A ecocardiografia é a melhor ferramenta para avaliar a função cardiovascular à cabeceira do doente de forma repetida, permitindo caracterizar melhor o desequilíbrio hemodinâmico, escolher adequadamente a opção terapêutica mais correcta e avaliar a resposta às intervenções realizadas. ${ }^{1}$ Este exame complementar deve ser sempre realizado em conjunto com a ecografia torácica, já que as informações recolhidas de ambos os exames se complementam, permitindo uma melhor acuidade clínica. ${ }^{8}$ No contexto de trauma abdominal e de suspeita de choque hemorrágico, a ecografia abdominal tem também um papel a desempenhar, dada a possibilidade de identificar precocemente coleções abdominais.

\section{Terapêutica de suporte}

A terapêutica de suporte tem por objectivo optimizar a perfusão tecidual, ${ }^{1}$ segundo as relações descritas para débito cardíaco, preenchimento e tónus vascular, contrabalançando os efeitos dos mecanismos fisiopatológicos na origem do choque e a progressiva falência dos mecanismos de compensação.

Seguindo a abordagem ABCDE, que pressupõe a estratégia 'reconhecer e tratar', a terapêutica de suporte deve ser iniciada logo que determinado problema passível de pôr em risco a vida do doente é identificado. ${ }^{6}$

No que se refere à terapêutica de suporte para o doente em choque, após a abordagem de A e de B no contexto da metodologia $A B C D E$ e não esquecendo os timings da terapêutica dirigida a cada entidade (que em algumas situações é emergente e idealmente realizada em simultâneo com a terapêutica de suporte) é de ponderar:

1 - Suplementação de oxigénio (nas suas diversas modalidades) ou ventilação assistida (invasiva ou não invasiva) de forma a optimizar a entrega celular de $\mathrm{O} 2^{1-5}$ : Nos doentes com alteração do estado de consciência, hipoven- tilação e movimentos respiratórios ineficazes, deve ser ponderada a ventilação mecânica invasiva com o objectivo de evitar tanto a hipoxemia e a hipercapnia como a hiperóxia e a hipocapnia. ${ }^{20}$ Nos restantes casos deve ser ponderada a suplementação de oxigénio, bem como a ventilação não invasiva, consoante a situação clínica do doente, mantendo os mesmos objectivos. ${ }^{20}$ É ainda de ter em conta que, ao diminuir o gasto de $\mathrm{O}_{2}$ pelo organismo, a sedação e o início de ventilação mecânica invasiva pode ter interesse apenas com este intuito. ${ }^{20}$

No contexto de compromisso do débito cardíaco e sinais de sobrecarga hídrica esquerda, a ventilação com pressão positiva, ao diminuir quer o retorno venoso, quer a pós-carga, melhora o débito cardíaco com consequentemente melhoria dos sinais de sobrecarga. 5,9-10

2 - Reforço do preenchimento vascular quando existe depleção intravascular ou quando existe benefício em aumentar as pressões nas cavidades cardíacas direitas, tendo em atenção que no contexto de choque hipovolémico por hemorragia, desde que se encontre disponível, o doente beneficia em primeiro lugar de suporte transfusional e que a infusão de grandes volumes de soros agrava a sua coagulopatia por diluição dos factores de coagulação. ${ }^{1-7,12}$

Preenchimento vascular - cristalóides (preferência pelas soluções equilibradas como o soro polielectrolítico ou o lactato de Ringer): bólus inicial de $5 \mathrm{ml} / \mathrm{kg}$ em $10 \mathrm{~min}$ (fluid challenge), seguido de titulação até aos $30 \mathrm{~mL} / \mathrm{kg}$ no contexto de sépsis, ou até ao efeito desejado vigiando os sinais de sobrecarga hídrica $(20-30 \mathrm{~mL} / \mathrm{kg}){ }^{2-4,27}$ No contexto de choque hemorrágico com hemorragia não controlada, limitar reposição hídrica, repetindo bólus de $5 \mathrm{~mL} / \mathrm{kg}$ com o objectivo de manter pulso radial palpável ou tensão arterial média da ordem dos $65 \mathrm{mmHg}$. Os objectivos tensionais devem, no entanto, ser individualizados consoante a situação clínica, nomeadamente no que se refere a doentes previamente hipertensos ou com trauma de crânio, que beneficiam de valores tensionais superiores. ${ }^{1,12}$

São considerados doentes com boa resposta a fluidos aqueles que apresentam uma subida imediata de 10\% $15 \%$ do volume sistólico ou do débito cardíaco após a administração de 250 - $500 \mathrm{~mL}$ de fluido. ${ }^{27}$ No contexto de sala de emergência, estes parâmetros podem ser avaliados directamente com recurso à ecocardiografia, ou por avaliação indirecta dos sinais clínicos a eles associados.

À partida, apenas os doentes que se encontram desde o início em sobrecarga hídrica esquerda não apresentam benefício potencial de preenchimento vascular, sendo que nos restantes casos a resposta a esta terapêutica deve ser avaliada (p. ex. através da administração de fluid challenge).

Preenchimento vascular-colóides: nos doentes em choque séptico com necessidade de grandes volumes de fluidos pode ser ponderada adicionalmente a administração de albumina, guiada pelo respectivo valor sérico (objectivo $>3 \mathrm{~g} / \mathrm{dL}){ }^{4}$ 
3 - Terapêutica vasoactiva: Suporte vasopressor no contexto de vasoplegia, ou de benefício potencial no aumento do retorno venoso; suporte inotrópico no contexto de compromisso do débito cardíaco. . $9-10^{-10}$

Suporte vasopressor - noradrenalina: terapêutica inicial em perfusão por via periférica até estabilidade clínica relativa (por exemplo veia proximal de grande calibre (14/16 gauge) e infusão por bomba infusora $10 \mathrm{mg} / 500 \mathrm{~mL}$ ). Seguidamente, perfusão por via central em seringa infusora titulando ritmo de $3 / 3$ minutos $(0,1-1,0 \mathrm{mcg} / \mathrm{kg} / \mathrm{min}) .^{3-5,13}$

Da revisão da literatura sobre este tema, é de referir que: a) em ambiente de unidade de cuidados intensivos, perfusões de noradrenalina na concentração de $65 \mathrm{mcg} / \mathrm{mL}$ (16 mg/250 mL) administrações por veia periférica durante uma média de 50 horas, e em dose máxima média de 0,7 $\mathrm{mcg} / \mathrm{kg} / \mathrm{min}$, foram seguras num universo de cerca de 700 doentes $^{18}$; b) em ambiente peri-operatório, perfusões de noradrenalina na concentração de $20 \mathrm{mcg} / \mathrm{mL}(10 \mathrm{mg} / 500$ $\mathrm{mL}$ ) foram seguras num universo de cerca de 14000 doentes $^{21}$; c) no que se refere aos casos em que existiram complicações derivadas da administração de noradrenalina, é de realçar que estas ocorreram em perfusões prolongadas (média de cerca de 55 horas), e em que $85 \%$ dos casos os acessos venosos utilizados eram distais em relação às fossas antecubital ou popliteia. ${ }^{22}$

Suporte inotrópico - inicialmente por via periférica de grande calibre, diluição em balão e infusão por bomba infusora, para que logo que se verifique estabilidade clínica relativa e colocação de cateter venoso central (CVC), se altere para infusão por seringa infusora em veia central titulando dose de 3/3 minutos:

- Opção A: dobutamina (efeito vasodilatador): diluição de $250 \mathrm{mg} / 50 \mathrm{~mL}$, para uma dose de $2,5-20 \mathrm{mcg} / \mathrm{kg} /$ $\min ^{5,8,13}$;

- Opção B: adrenalina (efeito vasopressor; não usar em monoterapia): diluição de $5 \mathrm{mg} / 50 \mathrm{~mL}$, para uma dose de 0,05 - 0,5 mcg/kg/min. .5,8,13

No que se refere ao choque distributivo, nomeadamente no contexto de sépsis, uma vez que predomina a vasoplegia, o fármaco vasoactivo inicial recomendado é a noradrenalina, devendo os restantes ser ponderados consoante a eventual necessidade crescente de dose..$^{2-4}$ Quanto ao choque cardiogénico, apesar da sua diferente etiologia, a recomendação é também de iniciar a terapêutica vasoactiva com noradrenalina, sendo no entanto provável a necessidade de a ela ser associada um inotrópico como a dobutamina ou a adrenalina. ${ }^{5}$ No que se refere aos restantes tipos de choque - não sendo os fármacos vasoactivos a primeira linha terapêutica - a noradrenalina pode eventualmente ser considerada - seja no choque hemorrágico como poupador de fluidos, ${ }^{23}$ seja no choque obstrutivo para promover o retorno venoso. ${ }^{24}$

4 - Protocolo hospitalar de transfusão maciça na abordagem inicial ao doente em choque hipovolémico por hemorragia major. ${ }^{12}$ No que se refere à aplicação do protocolo da Direcção Geral de Saúde da República Portuguesa ao contexto da avaliação do doente em choque hemorrágico na sala de emergência, é de destacar:

- Em caso de trauma ou hiperfibrinólise, administrar 1 $\mathrm{g}$ de ácido tranexâmico em 10 minutos, seguido de $1 \mathrm{~g}$ em perfusão durante oito horas. ${ }^{12}$

- Administrar 30 - 50 mg/kg de fibrinogénio enquanto se aguarda pela disponibilidade de outros produtos. ${ }^{12}$

- Se possível, guiar a administração de componentes sanguíneos por tromboelastometria rotacional. ${ }^{12}$

- Caso não exista tromboelastometria, a indicação inicial é para a administração de quatro unidades de concentrado eritrocitário + quatro unidades de plasma fresco congelado + uma pool de plaquetas; a reavaliar consoante os resultados analíticos e a evolução clínica. ${ }^{12}$

5 - Estratégias de resgate para doentes em peri-paragem ou com instabilidade hemodinâmica súbita, de onde se destaca a administração de vasopressores em bólus:

- Adrenalina (diluir $1 \mathrm{mg}$ em 20 -100 mL): bólus de $0.01 \mathrm{mg}$ (1 mL de uma solução $1 \mathrm{mg} / 100 \mathrm{~mL}$ ) repetidos de $1 / 1$ minuto ou bólus de $0,05 \mathrm{mg}(1 \mathrm{~mL}$ de uma solução de $1 \mathrm{mg} / 20 \mathrm{~mL}$ ) repetidos de $5 / 5$ minutos, titulando dose até resposta pretendida, seguido de lavagem da via após administração (bólus de $20 \mathrm{~mL}$ de soro por exemplo), administrados por via periférica proximal de grande calibre. ${ }^{14-17,25,26}$

\section{DISCUSSÃO}

O doente em choque é de difícil abordagem, pelo que deve ser sempre pedido apoio de maior diferenciação nomeadamente à medida que o quadro agrava e a necessidade de medidas de suporte de órgão aumenta.

O reconhecimento precoce do quadro pode ser dificultado em virtude da síndrome de sinais e sintomas apresentados pelo doente ser composto tanto pelos que são despoletados directamente pelo mecanismo fisiopatológico do choque, como pelos que são despoletados indirectamente pelos mecanismos fisiológicos de compensação, resultantes da resposta do sistema nervoso autónomo com activação do sistema nervoso simpático.

É comum que mecanismos de compensação sejam identificados erradamente como a causa do choque e não como uma resposta fisiológica, atrasando o diagnóstico correcto e a instituição de um plano terapêutico adequado.

Não são discutidas todas as alternativas existentes aos fármacos apresentados para cada situação clínica. Contudo, uma vez em que pode existir vantagem em as utilizar, a abordagem a este tema deve ser complementada com o recurso a outras fontes (é por exemplo de referir a possibilidade de recorrer à dopamina ou à terlipressina como vasopressor em determinados contextos, bem como à efedrina ou à fenilefrina como vasopressores de resgate, ou ainda recorrer ao levosimendan ou à milrinona como inotrópicos alternativos em caso de falência do ventrículo direito). Contudo, uma vez que estas não são nem as abordagens mais 
consensuais, nem as mais adaptadas ao ambiente de urgência, foi assumida a opção de não as aprofundar durante esta revisão.

A ecocardiografia é actualmente fundamental em ambiente de urgência na abordagem ao doente instável do ponto de vista hemodinâmico, permitindo dar resposta àquelas que são as dúvidas fundamentais neste contexto "Indicação para a administração de soros, vasopressor ou inotrópico?" - permitindo simultaneamente excluir diversas etiologias para o quadro de choque. Não sendo necessário nem desejável neste contexto a realização de um ecocardiograma formal, opta-se por destacar a abordagem proposta pela Sociedade Europeia de Cardiologia para a abordagem em ambiente de urgência/cuidados intermédios/ intensivos por não cardiologistas aos doentes com instabilidade hemodinâmica e que engloba a conjugação de dados da ecocardiografia transtorácica com dados da ecografia torácica que neste contexto deve ser sempre realizada. ${ }^{8}$

Existem actualmente alguns estudos que comprovam a segurança da administração de vasopressores e inotrópicos por via periférica. ${ }^{18,19,21}$ A colocação de um acesso venoso central num doente instável, tal como todos os procedimentos invasivos neste contexto, não é de todo ideal, podendo comprometer o prosseguir da reanimação do doente ao obrigar a equipa a centrar-se num procedimento específico.

Neste contexto, sugere-se que o doente seja sempre estabilizado previamente à realização de técnicas não emergentes, como a colocação de acessos centrais, optando inicialmente pela administração por via periférica quer de vasopressores, quer de inotrópicos, tendo em atenção que determinados procedimentos diminuem o risco desta administração - como são os referidos exemplos de escoIher uma veia periférica proximal de grande calibre, de optar pela diluição do fármaco num maior volume e pela sua consequente administração a um maior ritmo de perfusão, já que a grande maioria das complicações ocorre em perfusões prolongadas por acessos distais. ${ }^{22}$

É de notar que a administração de adrenalina por via endovenosa em bólus deve ser sempre realizada por equipas experientes na sua utilização. Contudo, a simplicidade e facilidade da sua administração, por oposição à preparação de uma perfusão, justifica que em determinadas situações clínicas, este possa ser o fármaco e a forma de administração ideais. ${ }^{14-17,25,26} \mathrm{~A}$ adrenalina diluída, e administrada em pequenos bólus de forma titulada, oferece a possibilidade de realizar uma terapêutica de ponte até ser possível administrar vasopressor/inotrópico através de uma perfusão.

Não foi revista neste trabalho a terapêutica dirigida em específico a cada entidade clínica. Contudo, esta é fulcral para o prognóstico do doente e idealmente seria posta em prática em simultâneo com terapêutica de suporte. É assim imprescindível não esquecer que, por exemplo, no contexto de choque hemorrágico ou séptico, o controlo cirúrgico do foco pode ser emergente, e que terapêuticas como a revascularização coronária no contexto de choque cardiogénico ou o início de antibioterapia na sépsis têm timings bem definidos. ${ }^{1-7}$

\section{CONCLUSÃO}

A abordagem ao doente em choque engloba o reconhecimento deste quadro clínico, o diagnóstico etiológico, a instituição de terapêutica de estabilização e a instituição de terapêutica dirigida, sendo seguida a metodologia $A B C D E$, o que significa que por vezes todos estes passos são realizados em simultâneo com o intuito de 'reconhecer e tratar'.

A instabilidade do doente admitido na sala de emergência condiciona a sua avaliação, já que a necessidade de implementação de medidas imediatas é condicionada pelas limitações logísticas impostas na colheita da história clínica, na realização de exames complementares de diagnóstico, na instituição de acessos venosos centrais ou de medidas invasivas de monitorização.

É importante reconhecer os sinais e sintomas acessíveis neste contexto - de onde se destaca a avaliação do débito cardíaco, tónus e preenchimento vascular - complementando a abordagem clínica com as informações recoIhidas com o apoio da ecografia e de outros exames complementares.

No que se refere à terapêutica de suporte inicial, devem ser consideradas estratégias adaptadas ao ambiente da sala de emergência como são a administração de fármacos vasoactivos por acesso venoso periférico, ou em bólus.

\section{NOTAS ADICIONAIS}

A pedido dos autores, o texto encontra-se redigido segundo a ortografia prévia ao novo acordo ortográfico.

\section{PROTECÇÃO DE PESSOAS E ANIMAIS}

Os autores declaram que os procedimentos seguidos estavam de acordo com os regulamentos estabelecidos pelos responsáveis da Comissão de Investigação Clínica e Ética e de acordo com a Declaração de Helsínquia da Associação Médica Mundial actualizada em 2013.

\section{CONFIDENCIALIDADE DOS DADOS}

Os autores declaram ter seguido os protocolos do seu centro de trabalho acerca da publicação de dados.

\section{CONFLITOS DE INTERESSE}

Os autores declaram não ter conflitos de interesse relacionados com o presente trabalho.

\section{FONTES DE FINANCIAMENTO}

Este projeto não recebeu qualquer apoio financeiro.

\section{REFERÊNCIAS}

1. Cecconi M, De Backer D, Antonelli M, Beale R, Bakker J, Hofer C, et al. Consensus on circulatory shock and hemodynamic monitoring. Task

force of the European Society of Intensive Care Medicine. Intensive Care Med. 2014;40:1795-815. 
2. Singer M, Deutschman CS, Seymour CW, Shankar-Hari M, Annane D, Bauer $\mathrm{M}$, et al. The third international consensus definitions for sepsis and septic shock (Sepsis-3). JAMA. 2016;315:801-10.

3. Esposito S, De Simone G, Boccia G, De Caro F, Pagliano P. Sepsis and septic shock: New definitions, new diagnostic and therapeutic approaches. J Glob Antimicrob Resist. 2017;10:204-12.

4. Rhodes A, Evans L, Alhazzani W, Levy MM, Antonelli M, Ferrer R. Surviving Sepsis Campaign: International Guidelines for Management of Sepsis and Septic Shock 2016. Crit Care Med. 2016;43:304-77.

5. Thiele H, Ohman EM, Desch S, Eitel I, de Waha S. Management of cardiogenic shock. Eur Heart J. 2015;36:1223-30.

6. Soar J, Nolan JP, Bottiger BW, Perkins GD, Lott C, Carli P, et al. European Resuscitation Council Guidelines for Resuscitation 2015, Section 3. Adult advanced life support. Resuscitation. 2015;95:100-47.

7. Ponikowski P, Voors AA, Anker SD, Bueno H, Cleland JG, Coats AJ, et al. 2016 ESC Guidelines for the Diagnosis and Treatment of Acute and Chronic Heart Failure: The Task Force for the Diagnosis and Treatment of Acute and Chronic Heart Failure of the European Society of Cardiology (ESC). Eur Heart J. 2016;37:2129-200.

8. Neskovic AN, Skinner H, Price S, Via G, De Hert S, Stankovic I, et al. Focus cardiac ultrasound core curriculum and core syllabus of the European Association of Cardiovascular Imaging. Eur Heart J Cardiovasc Imaging. 2018;19:475-81.

9. Steg G, James SK, Atar D, Badano LP, Blömstrom-Lundqvist C, Borger $M A$, et al. ESC guidelines for the management of acute myocardial infarction in patients presenting with ST-segment elevation. Eur Heart J. 2012;33:2569-619.

10. Roffi M, Patrono C, Collet JP, Mueller C, Valgimigli M, Andreotti F, et al. ESC guidelines for the management of acute coronary syndromes in patients without persistent ST-segment elevation. Eur Heart J. 2016;37:267-315.

11. Konstantinides SV, Torbicki A, Agnelli G, Danchin N, Fitzmaurice D, Galiè N, et al. 2014 ESC guidelines on the diagnosis and management of acute pulmonary embolism. Eur Heart J. 2014; 35:3033-69.

12. George F. Abordagem da transfusão maciça no adulto. Direcção Geral de Saúde da República Portuguesa. Norma nº 011/2013 de 30/07/2013 actualizada a 18/07/2017 [consultado 2019 nov 20]. Disponível em: https://www.dgs.pt/?cr=24597.

13. Tarvasmak T, Lassus J, Varpula M, Sionis A, Sund R, Køber L, et al. Current real-life use of vasopressor and inotropes in cardiogenic shock adrenaline use is associated with excess organ injury and mortality. Crit Care Med. 2016;20:208.

14. Weingart S. Push-dose pressors for immediate blood pressure control. Clin Exp Emerg Med. 2015;2:131-2.

15. Gottlieb M. Bolus dose of epinephrine for refractory post-arrest hypotension. CJEM. 2018;20:S9-13.

16. Acquisto NM, Bodkin RP, Johnstone C. Medication errors with push dose pressors in the emergency department and intensive care units. Am J Emerg Med. 2017;35:1964-5.

17. Tilton LJ, Eginger $\mathrm{KH}$. Utility of push-dose vasopressors for temporary treatment of hypotension in the emergency department. J Emerg Nurs. 2016;42:279-81.

18. Cardenas-Garcia J, Schaub KF, Belchikov YG, Narasimhan M, Koenig SJ, Mayo PH. Safety of peripheral intravenous administration of vasoactive medication. J Hosp Med. 2015;10:581-5.

19. Patregnani JT, Sochet AA, Klugman D. Short-term peripheral vasoactive infusions in pediatrics: where is the harm? Pediatr Crit Care Med 2017;18:e378-81

20. Nolan JP, Soar J, Cariou A, Cronberg T, Moulaert VR, Deakin CD, et al. European Resuscitation Council and European Society of Intensive Care Medicine Guidelines for Post-resuscitation Care 2015: Section 5 of the European Resuscitation Council Guidelines for Resuscitation 2015. Resuscitation. 2015;95:202-22.

21. Pancaro C, Shah N, Pasma W, Saager L, Cassidy R, van Klei W, et al. Risk of major complications after perioperative norepinephrine infusion through peripheral intravenous lines in a multicenter study. Anesth Analg. 2019;131:1060-5.

22. Loubani OM, Green RS. A systematic review of extravasation and local tissue injury from administration of vasopressors through peripheral intravenous catheters and central venous catheters. J Crit Care 2015;30:653.e9-17.

23. Dunberry-Poissant S, Gilbert K, Bouchard C, Baril F, Cardinal AM, L'Ecuyer S, et al. Fluid sparing and norepinephrine use in a rat model of resuscitated haemorrhagic shock: end-organ impact. Intensive Care Med Exp. 2018;6:47.

24. Zhang $H$, Spapen $H$, Vincent JL. Effects of dobutamine and norepinephrine on oxygen availability in tamponade-induced stagnant hypoxia: a prospective, randomized, controlled study. Crit Care Med. 1994;22:299-305.

25. Holden D, Ramich J, Timm E, Pauze D, Lesar T. Safety considerations and guideline-based safe use recommendations for "bolus-dose" vasopressors in the emergency department. Ann Emerg Med. 2018;71:83-92.

26. Cole JB. Bolus-dose vasopressors in the emergency department: first, do no harm; second, more evidence is needed. Ann Emerg Med. 2018;71:93-5.

27. Roger C, Zieleskiewicz L, Demattei C, Lakhal K, Piton G, Louart B, et al. Time course of fluid responsiveness in sepsis: the fluid challenge revisiting (FCREV) study. Crit Care. 2019;23:179. 